\title{
Metabolic Engineering of the Methylotrophic Yeast Pichia Pastoris (Komagataella phaffii) for the Production of B-alanine From Methanol
}

\author{
Liangtian Miao \\ Institute of Microbiology Chinese Academy of Sciences \\ Yin Li ( $\square$ yli@im.ac.cn ) \\ Institute of Microbiology Chinese Academy of Sciences \\ Taicheng Zhu \\ Institute of Microbiology Chinese Academy of Sciences
}

\section{Research}

Keywords: Pichia pastoris (Komagataella phaffii), $\beta$-alanine (3-aminopropionic acid), methanol, aspartate decarboxylation, aspartate dehydrogenase

Posted Date: June 29th, 2021

DOl: https://doi.org/10.21203/rs.3.rs-651971/v1

License: (c) (i) This work is licensed under a Creative Commons Attribution 4.0 International License. Read Full License 


\section{Abstract}

$\beta$-Alanine (3-aminopropionic acid), is the only naturally occurring $\beta$-amino acid and an important precursor for the synthesis of a variety of nitrogen-containing chemicals. Fermentative production of $\beta$ alanine from renewable feedstocks such as glucose has attracted significant interest in recent years. Methanol has become an emerging and promising renewable feedstock for biomanufacturing as an alternative to glucose. In this work, we demonstrated the feasibility of $\beta$-alanine production from methanol using Pichia pastoris (Komagataella phaffii) as a methylotrophic cell factory. Aspartate decarboxylases (ADCs) from different sources were screened and expressed in P. pastoris, followed by the optimization of aspartate decarboxylation by increasing the ADC copy number and $C 4$ precursor supply via the overexpression of aspartate dehydrogenase. The production potential of the best strain was further evaluated in a 1-liter fermenter, and a $\beta$-alanine titer of $5.6 \mathrm{~g} / \mathrm{L}$ was obtained. To our best knowledge, this is the highest chemical production titer ever reached in $P$. pastoris using methanol as the substrate.

\section{Introduction}

$\beta$-Alanine (3-aminopropionic acid) is a naturally occurring $\beta$-amino acid that serves as a precursor for the biosynthesis of a variety of nitrogen-containing chemicals, such as D-pantothenic acid (vitamin B5) (Tigu et al. 2018), coenzyme A (CoA) (Tomita et al. 2014), carnosine (Harris et al. 2006; Sale et al. 2010), and poly-alanine (nylon-3) (Steunenberg et al. 2013). $\beta$-Alanine can be chemically synthesized via the ammonification of acrylonitrile (Carlson 1943), hydroxylation of $\beta$-aminopropionitrile in the presence of barium hydroxide (Ford 1945), or the reaction of acrylic acid with ammonium carbonate and $\mathrm{CO}_{2}$ (Ohara et al. 2011). However, these chemical synthesis routes are not sustainable due to the use of nonrenewable substrates and harsh reaction conditions, and biological processes for $\beta$-alanine synthesis have gained increasing interest.

Biological production of $\beta$-alanine is achieved through biotransformation or fermentation. In the biotransformation route, $\beta$-alanine can be directly synthesized from L-aspartic acid via decarboxylation by L-aspartate-a-decarboxylase (ADC, EC: 4.1.1.11) (Li et al. 2018; Pei et al. 2017; Shen et al. 2014), or indirectly synthesized from fumaric acid as a cheaper substrate, via the consecutive action of aspartate ammonia-lyase (AspA, EC 4.3.1.1) and ADC (Qian et al. 2018; Wang et al. 2020). Although a very high $\beta$ alanine titer (up to $200 \mathrm{~g} / \mathrm{L}$ ) has been reached with whole-cell transformation, the process is not entirely sustainable due to the use of expensive precursors or petrochemicals (i.e., L-aspartic acid or fumaric acid) as substrates. Consequently, the fermentation route has been pursued to synthesize $\beta$-alanine from renewable feedstocks. The fermentative production of $\beta$-alanine from glucose has been described in several reports on the metabolic engineering of Escherichia coli, and promising $\beta$-alanine titers ranging from 32.3 to $43.12 \mathrm{~g} / \mathrm{L}$ were obtained in these studies (Piao et al. 2019; Song et al. 2015; Zou et al. 2020).

Although glucose is the most widely used raw material for the production of biochemicals, the exploration of alternative feedstocks has remained a central task in the research on 
biomanufacturing (Liu et al. 2021). Methanol is considered one of the most promising feedstocks due to its unique advantages such as providing highly reduced carbon, not competing with food sources, and potentially sustainable production in the future (Zhu et al. 2020). Consequently, there is an emerging trend of utilizing methanol as an alternative feedstock for chemical production using natural or synthetic methylotrophs (Guo et al. 2021; Jin et al. 2021; Tuyishime et al. 2018; Whitaker et al. 2017; Zhu et al. 2016).

In this work, we aimed to achieve the production of $\beta$-alanine from methanol using Pichia pastoris (Komagataella phaffii) as the methylotrophic cell chassis (Fig. 1). ADCs from different sources were screened and expressed in $P$. pastoris, followed by the optimization of aspartate decarboxylation and C4 precursor supply, and the best strain reached a $\beta$-alanine titer of $5.6 \mathrm{~g} / \mathrm{L}$. To our best knowledge, this is the first report demonstrating the feasibility of using $P$. pastoris as the chassis for the production of amino acids from methanol.

\section{Materials And Methods}

\section{Strains, media, and growth conditions}

The primers, strains and plasmids used in this study are listed in Table 1 and Table 2. For E. coli strain construction, the cells were cultured aerobically at $37^{\circ} \mathrm{C}$ in Luria broth $(10 \mathrm{~g} / \mathrm{L}$ tryptone, $5 \mathrm{~g} / \mathrm{L}$ yeast extract, and $10 \mathrm{~g} / \mathrm{L} \mathrm{NaCl})$. For the construction of $P$. pastoris strains, YPD $(20 \mathrm{~g} / \mathrm{L}$ peptone, $10 \mathrm{~g} / \mathrm{L}$ yeast extract and $20 \mathrm{~g} / \mathrm{L}$ glucose) or MD ( $20 \mathrm{~g} / \mathrm{L}$ glucose, $13.4 \mathrm{~g} / \mathrm{L}$ yeast nitrogen base, $0.4 \mathrm{mg} / \mathrm{L}$ biotin) medium was used, and the cells were cultured aerobically at $30^{\circ} \mathrm{C}$. Kanamycin $(50 \mathrm{mg} / \mathrm{L}), \mathrm{G} 418(200$ $\mathrm{mg} / \mathrm{L})$, zeocin (50 mg/L) or hygromycin (50 $\mathrm{mg} / \mathrm{L})$ was added where appropriate.

\section{Construction of recombinant plasmids}

The P. pastoris HIS4 gene was amplified by PCR from pAO and ligated into the BamHI site of pPICZA to generate the expression vector pMPICZHis. The zeocin resistance marker of pMPICZHis was replaced with the kanamycin or hygromycin resistance gene using a Gibson assembly strategy to yield the expression vectors pMPICKmHis and pMPIC2H, respectively. The panD genes from $B$. paralicheniformis ATCC 9945a, B. subtilis, S. griseorubiginosus, and C. glutamicum, the aspDH genes from Ochrobactrum anthropi ATCC 4918 and Serratia proteamaculans, as well as the gene encoding cysteine sulfinic acid decarboxylase from $T$. castaneum were codon optimized, synthesized and subcloned into pMPICZHis between the BstBI and Notl sites. The genes encoding cytosolic and mitochondrial aspartate aminotransferases were PCR amplified from genomic DNA of $P$. pastoris GS115 and cloned into pMPICKHis between the BstBI and Notl sites. The ppc and pck genes from E. coli, as well as the pck gene from Actinobacillus succinogenes were PCR amplified and cloned into pMPICKmHis between the BstBI and Notl sites.

A previously described multi-copy plasmid construction method (Yu et al. 2020a) was applied for the construction of the 2-, or 3-gene co-expression plasmids. The pMPICZHis-gene plasmid was double- 
digested with $S p h l$ and $B a m H I$ to generate the expression cassette, which was then inserted between the $S p h l$ and Bg/ll sites of pMPICZHis-gene to create pMPICZHis-2genes. Similarly, the gene cassette was reinserted between the $S p h l$ and $B g$ Il sites of pMPICZHis-2genes to create pMPICZHis-3genes.

\section{Construction of recombinant strains}

The KU70 gene of the wild-type P. pastoris strain GS115 was deleted using the Cre-LoxP method to improve the homologous recombination efficiency (Guo et al. 2021; Weninger et al. 2018). The expression vectors pMPICZHis-bliPanD, pMPICZHis-bsPanD, pMPICZHis-sgePanD, pMPICZHis-cguPanD, pMPICZHisTcCSADC, pMPICZHis-ADC-Oan, pMPICZHis-ADC-Spe, pMPICZHis-2ADC, and pMPICZHis-2ADC-Spe were linearized with $B s p E l$ and used to transform the $\triangle \mathrm{ku} 70$ strain by electroporation. Positive transformants were screened on MD plates and named BliADC, BsADC, SgeADC, CguADC, TcCSADC, ADC-Oan, ADC-Spe, $2 A D C$ and $2 A D C-S p e$, respectively. The expression vectors pMPICKmHis-AAT1 and pMPICKmHis-AAT2 were linearized using $B s p E l$ and used to transform the BsADC strain to generate ADC-AAT1 and ADCAAT2, respectively. The expression vectors pMPIC2H-PPC, pMPIC2H-AsPCK, and pMPIC2H-PCK were linearized with BspEI, Stul and Sspl, respectively, and used to transform the strain 2ADC-Spe, yielding the recombinant strains PPC, AsPCK and PCK, respectively.

\section{$\beta$-Alanine production in shake-flask fermentations}

The strains were precultured in YPD medium and transferred to $25 \mathrm{~mL}$ of BMMY medium $(20 \mathrm{~g} / \mathrm{L}$ peptone, $10 \mathrm{~g} / \mathrm{L}$ yeast extract, $13.4 \mathrm{~g} / \mathrm{L}$ YNB, $0.4 \mathrm{mg} / \mathrm{L}$ biotin, $8.7 \mathrm{~g} / \mathrm{L}$ monobasic potassium phosphate, $\mathrm{pH}$ 6.0) in $250 \mathrm{~mL}$ baffled shake flasks and cultured at $30^{\circ} \mathrm{C}$ and $220 \mathrm{rpm}$. Recombinant gene expression was induced by adding $200 \mu \mathrm{l}$ of pure methanol to each flask, followed by feeding with $200 \mu \mathrm{l}$ of methanol at $12 \mathrm{~h}$ intervals.

\section{$\beta$-Alanine production by fed-batch fermentation in a 1-liter fermenter}

The strains were first grown in $50 \mathrm{~mL}$ YPD for $36 \mathrm{~h}$ and transferred into 1-liter stirred tank reactors (Infors, Switzerland) containing $0.8 \mathrm{~L}$ of BMGY $(20 \mathrm{~g} / \mathrm{L}$ peptone, $10 \mathrm{~g} / \mathrm{L}$ yeast extract, $13.4 \mathrm{~g} / \mathrm{L}$ YNB, $0.4 \mathrm{mg} / \mathrm{L}$ biotin, $8.7 \mathrm{~g} / \mathrm{L}$ monobasic potassium phosphate, $40 \mathrm{~g} / \mathrm{L}$ glycerol, $\mathrm{pH}$ 6.0) supplemented with $4.0 \mathrm{~mL}$ PTM1 trace salts $\left(6 \mathrm{~g} / \mathrm{L} \mathrm{CuSO}_{4} \cdot 5 \mathrm{H}_{2} \mathrm{O}, 0.09 \mathrm{~g} / \mathrm{L} \mathrm{KI}, 3 \mathrm{~g} / \mathrm{L} \mathrm{MnSO}_{4} \cdot \mathrm{H}_{2} \mathrm{O}, 0.02 \mathrm{~g} / \mathrm{L} \mathrm{H}_{3} \mathrm{BO}_{3}, 0.2 \mathrm{~g} / \mathrm{L}\right.$ $\mathrm{MoNa}_{2} \mathrm{O}_{4} \cdot 2 \mathrm{H}_{2} \mathrm{O}, 0.5 \mathrm{~g} / \mathrm{L} \mathrm{CoCl}_{2}, 20 \mathrm{~g} / \mathrm{L} \mathrm{ZnCl}_{2}, 65 \mathrm{~g} / \mathrm{L} \mathrm{FeSO}_{4} \cdot 7 \mathrm{H}_{2} \mathrm{O}, 0.2 \mathrm{~g} / \mathrm{L}$ biotin, $\left.5.0 \mathrm{~mL} / \mathrm{L} \mathrm{H}_{2} \mathrm{SO}_{4}\right)$. The temperature was set to $30^{\circ} \mathrm{C}$, the pH was controlled at 6.0 by adding $\mathrm{NH}_{3} \cdot \mathrm{H}_{2} \mathrm{O}(28 \%$, v/v), the dissolved oxygen concentration was kept above $20 \%$ of the atmospheric value by varying the air flow rate between 0.5 and $2 \mathrm{~L} / \mathrm{min}$. The entire cultivation started with a batch phase lasting for 20-24 h. Heterologous gene expression was induced by the addition of $0.25 \%(\mathrm{v} / \mathrm{v})$ methanol, and the methanol concentration was maintained at $3 \mathrm{~g} / \mathrm{L}$ throughout the entire fermentation span using an automatic methanol control station (FC2002, East China University of Science and Technology, Shanghai, China).

\section{Analytical methods}


The cell growth was analyzed by measuring the optical density at $600 \mathrm{~nm}$. $\beta$-Alanine production was measured by high-performance liquid chromatography (HPLC) with a variable wavelength detector (VWD) set to $334 \mathrm{~nm}$ and an Agilent ZRABOX SB-C18 column $(4.6 \mathrm{~mm} \times 250 \mathrm{~mm}, 5 \mu \mathrm{m})$ after centrifugation of the fermentation samples and o-phthalaldehyde (OPA) derivatization (Pei et al. 2017). The mobile phase consisted of $35 \mathrm{mM}$ sodium acetate $(\mathrm{pH} 7.5)$ containing $30 \%$ methanol with a flow rate of $1 \mathrm{~mL} / \mathrm{min}$.

\section{Results}

\section{Overexpression of aspartate- $\alpha$-decarboxylases (ADCs) from different sources for the production of $\beta$ - alanine and tolerance of the $P$. pastoris chassis to $\beta$-alanine}

Since aspartate is the direct precursor for $\beta$-alanine synthesis via decarboxylation by ADCs, and was reported to have one of the largest precursor pool sizes among all amino acids in $P$. pastoris (Carnicer et al. 2012), we hypothesized that overexpression of the ADC gene would lead to the accumulation of $\beta$ alanine. Four genes encoding aspartate-a-decarboxylase from B. paralicheniformis, B. subtilis, $S$. griseorubiginosus, $C$. glutamicum, as well as the gene encoding cysteine sulfinic acid decarboxylase from T. castaneum, which was reported to possess higher decarboxylation activity than ADCs, were evaluated in this work. The coding sequences were individually placed under the control of the strong AOX1 promoters and integrated into the genome of $P$. pastoris. In order to increase the efficiency of homologous recombination (HR), the KU70 mutant strain ( $\triangle$ ku70) which has impaired nonhomologous end joining (NHEJ) was used as a parent strain (Guo et al. 2021; Weninger et al. 2018). The individual overexpression of ADCs from B. paralicheniformis, B. subtilis and $C$. glutamicum enabled the accumulation of $\beta$-alanine after $6 \mathrm{~d}$ of fermentation when feeding the strains with methanol (Fig. 2). The highest titer (658.9 mg/L) was reached by the recombinant strain expressing B. subtilis ADC (BsADC) (Fig. 2), and BsADC was used as the starting strain for further metabolic engineering. To investigate whether the tolerance to $\beta$-alanine was a limiting factor for $\beta$-alanine production, P. pastoris was cultivated in media supplemented with different concentrations of $\beta$-alanine. The results showed that yeast cell growth was substantially reduced only in the presence of $80 \mathrm{~g} / \mathrm{L}$ of $\beta$-alanine (Fig. 3).

\section{Overexpression of aspartate transferase (AAT) or aspartate dehydrogenase (AspDH) to increase aspartate supply for improved $\beta$-alanine synthesis}

The conversion of oxaloacetate (OAA) into aspartate can be achieved by the transfer of an amino group from glutamate catalyzed by aspartate aminotransferase (AAT), or direct amination with ammonium as the amino group donor catalyzed by aspartate dehydrogenase (AspDH). P. pastoris possesses a mitochondrial AAT (encoded by AAT1) and a cytosolic AAT (AAT2), and both genes were cloned into the AOX1 vector and tested for their effects on $\beta$-alanine synthesis, respectively. In a previous paper, we reported that an AspDH from S. proteamaculans has high activity and stability (Li et al. 2017a). Thus, the SpeAspDH was also overexpressed in recombinant $P$. pastoris using the same strategy as AAT1 and AAT2. The shake-flask fermentation results showed that while neither AAT1 or AAT2 significantly 
improved $\beta$-alanine synthesis, the $\beta$-alanine titer was increased by $19.6 \%$ (to $787.9 \mathrm{mg} / \mathrm{L}$ ) by overexpressing SpeAspDH (Fig. 4).

\section{Further improvement of $\beta$-alanine production by increasing the ADC copy number}

After optimization of the aspartate precursor supply, we investigated whether aspartate decarboxylation is a potential bottleneck for $\beta$-alanine production by increasing the copy number of the encoding gene. Vectors harboring two copies of the ADC expression cassette alone and together with the SpeAspDH expression cassette were introduced into the $\triangle$ ku70 strain, resulting in the recombinant strains $2 A D C$ and 2ADC-Spe, respectively. The multi-copy strains $2 A D C$ and $2 A D C$-Spe exhibited 53.9 and $52.6 \%$ increases of $\beta$-alanine production compared with their respective single-copy counterparts, ADC and ADC-Spe (Fig. 5). However, the cell growth of $2 A D C$ and $2 A D C$-Spe was significantly slower than that of the wild-type strain, illustrating the negative effects of $A D C$ overexpression on the physiology of yeast cells.

\section{Effect of strengthening phosphoenolpyruvate (PEP) carboxylation on $\beta$-alanine production}

A number of studies showed that $\mathrm{CO}_{2}$ fixation-based carboxylation of $\mathrm{C} 3$ metabolites plays an important role in the synthesis of $\mathrm{C} 4$ precursors (Tan et al. 2013). Therefore, the effect of strengthening PEP carboxylation on $\mathrm{C} 4$ precursor supply and thereby $\beta$-alanine synthesis was further investigated. Since PEP can be converted into OAA by either PEP carboxylase (PPC) or PEP carboxykinase (PCK), the encoding $p p c$ and $p c k$ genes from E. coli, as well as pckfrom A. succinogenes (Hu et al. 2018) were individually overexpressed in $2 A D C$-Spe to evaluate their impact on $\beta$-alanine production in shake-flask fermentations. However, only a marginal (6.7\%) increase of the $\beta$-alanine titer was observed in the $p p c$ overexpressed strain compared to the control (2ADC-Spe), while both PCKs from A. succinogenes and $E$. coli decreased the $\beta$-alanine titer (by 13.2 and 7.5\%, respectively) (Fig. 6).

\section{Fed-batch fermentation to improve $\beta$-alanine production}

In order to obtain a higher $\beta$-alanine titer, fed-batch fermentation of the 2ADC-Spe strain was performed in a $1 \mathrm{~L}$ fermenter using a two-stage strategy. The fermentation was started with a glycerol phase $(40 \mathrm{~g} / \mathrm{L}$ glycerol) to facilitate biomass accumulation. When the glycerol was depleted and an $\mathrm{OD}_{600}$ of approximately 80 was reached, the methanol phase was induced by maintaining the methanol concentration at $3 \mathrm{~g} / \mathrm{L}$ using an on-line methanol analyzer. $\beta$-Alanine accumulation began in the methanol phase, and the highest $\beta$-alanine titer reached $5.6 \mathrm{~g} / \mathrm{L}$ (Fig. 7). However, the yeast cells grew very slowly during the entire methanol phase and the biomass only reached an $\mathrm{OD}_{600}$ of 123 after $106 \mathrm{~h}$ (Fig. 7), corresponding to an average specific growth rate of $0.0044 \mathrm{~h}^{-1}$.

\section{Discussion}

Pichia pastoris is one of the most widely used eukaryotic expression systems for heterologous proteins, and in recent years, its potential as a cell factory for the production of chemicals is also receiving increasing attention (Zhu et al. 2019). As a native methylotroph, P. pastoris has unique advantages over 
S. cerevisiae and E. coli when methanol is used as a feedstock. Nevertheless, it is still challenging to engineer $P$. pastoris for the production of chemicals, largely because as a Crabtree-negative yeast, $P$. pastoris tends to accumulate biomass rather than produce metabolites. Reports on metabolite production in fermentations of engineered $P$. pastoris mainly remain at the proof-of-concept stage, with titers usually lower than $1 \mathrm{~g} / \mathrm{L}$, especially when methanol is used as substrate (Gao et al. 2021). Several recent studies reported gram per liter metabolites production in shake flasks, with examples including isobutanol (Siripong et al. 2020; Siripong et al. 2018), malic acid (Guo et al. 2021), and D-lactic acid (Yamada et al. 2019). Unfortunately, the chemicals production potential of these recombinant $P$. pastoris strains was not fully evaluated by cultivation on the fermenter scale. In this study, we achieved a $\beta$-alanine titer of $1.7 \mathrm{~g} / \mathrm{L}$ in shake flasks, and the production of the strain was further evaluated in a 1-liter fermenter using a two-stage strategy with a high initial biomass. Finally, $5.6 \mathrm{~g} / \mathrm{L}$ of $\beta$-alanine titer was obtained, which is the highest chemical production titer ever reached in $P$. pastoris using methanol as the substrate.

Aspartate decarboxylation is the most important step of $\beta$-alanine synthesis. Although ADCs from different sources have been evaluated for $\beta$-alanine synthesis in E. coli (Feng et al. 2019; Liu et al. 2019; Pei et al. 2017; Song et al. 2015), the protein expression levels and enzyme activities of these ADCs may be different in eukaryotic hosts such as P. pastoris. Accordingly, we evaluated bacterial ADCs from different sources, including ones known to function well in E. coli such as the ADCs from C. glutamicum (Song et al. 2015) and B. subtilis (Pei et al. 2017), as well as the recently reported insect TCCSADC, which is reported to be a dimer that is resistant to turnover-dependent inactivation observed in ADCs from prokaryotes (Liu et al. 2019; Yu et al. 2020b). Only ADCs from Bacillus spp. exhibited high activity in the $P$. pastoris system, and the mere expression of $B$. subtilis ADC resulted in an initial success in $\beta$-alanine accumulation. Moreover, we showed that $\beta$-alanine production can be remarkably improved by doubling the ADC copy number, indicating that even with the strong AOX1 promoter, the decarboxylation of aspartate still remains the bottleneck for $\beta$-alanine synthesis. Increasing the copy number of the target gene is a widely used strategy in recombinant protein expression in P. pastoris (Yu et al. 2020a). Our work suggested that this strategy is still important in tuning metabolic pathways for chemical synthesis.

Increasing the aspartate supply is also crucial for achieving high $\beta$-alanine production. To this end, two strategies were applied. First, we aimed at increasing the conversion of OAA into aspartate by screening for appropriate enzymes for reductive amination. Although the overexpression of aspartate aminotransferase (AAT) was widely used to increase aspartate flux for the production of aspartate family amino acids (AFAAs) in industrial microbes such as C. glutamicum and E. coli (Li et al. 2017; Piao et al. 2019), the efficacy of AspDH overexpression for enhancing AFAAs production was rarely. The present study demonstrates that the overexpression of SpeAspDH can significantly increase $\beta$-alanine production, corroborating the potential of AspDHs for the production of AFAAs. The second strategy is to increase OAA supply by strengthening C3 carboxylation. PPC exhibits high affinity for bicarbonate and high catalytic velocity in the carboxylation of PEP, but the energy contained in PEP is lost in this reaction with the release of inorganic phosphate. Conversely, PCK can conserve the high energy of PEP, leading to net production of ATP for growth and cell maintenance, but it has low affinity for bicarbonate and relatively 
low catalytic velocity (Tan et al. 2013). In this study, the overexpression of EcPPC only led to a slight increase of the $\beta$-alanine titer, and pck overexpression even decreased $\beta$-alanine production, suggesting that OAA supply may not be the bottleneck for $\beta$-alanine synthesis at the current stage, and there might be other rate-limiting factors that should be resolved in future studies.

Another noteworthy phenomenon observed this work is that the growth of the cells was remarkably decreased, especially for strains harboring two copies of ADC. After shifting to the methanol phase in the fermenter, the yeast cells grew very slowly, with an average specific growth rate of $0.0044 \mathrm{~h}^{-1}$, which was an order of magnitude lower than that of the wild-type strain under the same conditions (usually more than $0.04 \mathrm{~h}^{-1}$ ). The impaired cell growth of the ADC-expressing strain is less likely caused by product inhibition, because the $\beta$-alanine titer far below its inhibitory concentration. Nevertheless, this phenomenon is not quite unexpected because as an important metabolic intermediate, aspartate takes part in many biological processes, such as the synthesis of AFAAs (Li et al. 2017b; Park and Lee 2010), protecting microbes against acid stress (Wu et al. 2013), shuttling of redox equivalents (Bakker et al. 2001), etc., and depletion of aspartate for $\beta$-alanine synthesis may cause severe perturbations of the normal physiology of yeast cells. The underlying mechanism is currently under investigation using omics approaches.

\section{Conclusions}

Recombinant $P$. pastoris strains were constructed for the production of $\beta$-alanine from methanol by screening and overexpressing ADCs from different sources. The $\beta$-alanine titer was further increased by increasing ADC copy number and overexpression of AspDH and PPC. The production potential of the best producing strain was evaluated on 1-liter fermenter and $5.6 \mathrm{~g} / \mathrm{L}$ of $\beta$-alanine titer was obtained, which is highest chemical production titer ever reached in $P$. pastoris using methanol as the substrate. This work is the first attempt to produce amino acids from methanol using recombinant $P$. pastoris as the cell chassis.

\section{Abbreviations}

ADC: aspartate-a-decarboxylases

AspA: aspartate ammonia-lyase

AAT: aspartate transferase

AspDH: aspartate dehydrogenase

HR: homologous recombination

NHEJ: nonhomologous end joining

OAA: oxaloacetate 
PEP: phosphoenolpyruvate

PPC: PEP carboxylase

PCK: PEP carboxykinase

AFAAs: aspartate family amino acids

\section{Declarations}

Acknowledgements

Not applicable.

\section{Funding}

This work was supported by the National Key R\&D Program of China (2018YFA0901400) and the National Natural Science Foundation of China (No. 31970039)

\section{Author information}

Affiliations

CAS Key Laboratory of Microbial Physiological and Metabolic Engineering, State Key Laboratory of Microbial Resources, Institute of Microbiology, Chinese Academy of Sciences, Beijing 100101, People's Republic of China

Liangtian Miao, Yin Li \& Taicheng Zhu

University of Chinese Academy of Sciences, Beijing 100049, People's Republic of China

Liangtian Miao

\section{Contributions}

TCZ and YL conceived the study and revised the manuscript. LTM designed and carried out the experiments, and drafted the manuscript. All authors read and approved the final manuscript.

Corresponding author

Correspondence to Yin Li \& Taicheng Zhu

Availability of data and materials

All data generated or analyzed during this study are included in this article. 
Ethics approval and consent to participate

Not applicable.

\section{Consent for publication}

All authors approved the consent for publishing the manuscript to Bioresources and Bioprocessing.

\section{Competing interests}

The authors declare that they have no competing interests.

\section{References}

Bakker BM, Overkamp KM, van Maris AJ, Kötter P, Luttik MA, van Dijken JP, Pronk JT (2001)

Stoichiometry and compartmentation of NADH metabolism in Saccharomyces cerevisiae. FEMS Microbiol Rev 25(1):15-37. https://doi.org/10.1111/j.1574-6976.2001.tb00570.x

Carlson GH (1943) Preparation of beta-alanine. US Patents 2,336,067, 7 Dec. 1943.

Carnicer M, Canelas AB, Ten Pierick A, Zeng Z, van Dam J, Albiol J, Ferrer P, Heijnen JJ, van Gulik W (2012) Development of quantitative metabolomics for Pichia pastoris. Metabolomics 8(2):284-298. https://doi.org/10.1007/s11306-011-0308-1

Feng Z, Zhang J, Chen G, Ge Y, Zhang X, Zhu H (2019) Extracellular Expression of L-aspartate-alphadecarboxylase from Bacillus tequilensis and its application in the biosynthesis of beta-alanine. Appl Biochem Biotechnol 189(1):273-283. https://doi.org/10.1007/s12010-019-03013-1

Ford JH (1945) The alkaline hydrolysis of $\beta$-aminopropionitrile. Journal of the American Chemical Society 67(5):876-877. https://doi.org/10.1021/ja01221a503

Gao J, Jiang L, Lian J (2021) Development of synthetic biology tools to engineer Pichia pastoris as a chassis for the production of natural products. Synth Syst Biotechnol 6(2):110-119. https://doi.org/10.1016/j.synbio.2021.04.005

Guo F, Dai Z, Peng W, Zhang S, Zhou J, Ma J, Dong W, Xin F, Zhang W, Jiang M (2021) Metabolic engineering of Pichia pastoris for malic acid production from methanol. Biotechnol Bioeng 118(1):357371. https://doi.org/10.1002/bit.27575

Harris RC, Tallon MJ, Dunnett M, Boobis L, Coakley J, Kim HJ, Fallowfield JL, Hill CA, Sale C, Wise JA (2006) The absorption of orally supplied beta-alanine and its effect on muscle carnosine synthesis in human vastus lateralis. Amino Acids 30(3):279-89. https://doi.org/10.1007/s00726-006-0299-9 
Hu G, Zhou J, Chen X, Qian Y, Gao C, Guo L, Xu P, Chen W, Chen J, Li Y, Liu L (2018) Engineering synergetic $\mathrm{CO}_{2}$-fixing pathways for malate production. Metab Eng 47:496-504.

https://doi.org/10.1016/j.ymben.2018.05.007

Jin X, Zhang W, Wang Y, Sheng J, Xu R, Li J, Du G, Kang Z (2021) Biosynthesis of non-animal chondroitin sulfate from methanol using genetically engineered Pichia pastoris. Green Chemistry. https://doi.org/10.1039/d1gc00260k

Li H, Lu X, Chen K, Yang J, Zhang A, Wang X, Ouyang P (2018) $\beta$-alanine production using whole-cell biocatalysts in recombinant Escherichia coli. Molecular Catalysis 449:93-98. https://doi.org/10.1016/j.mcat.2018.02.008

Li H, Zhu T, Miao L, Zhang D, Li Y, Li Q, Li Y (2017a) Discovery of novel highly active and stable aspartate dehydrogenases. Sci Rep 7(1):7881. https://doi.org/10.1038/s41598-017-05522-7

Li Y, Wei H, Wang T, Xu Q, Zhang C, Fan X, Ma Q, Chen N, Xie X (2017b) Current status on metabolic engineering for the production of I-aspartate family amino acids and derivatives. Bioresour Technol 245(Pt B):1588-1602. https://doi.org/10.1016/j.biortech.2017.05.145

Liu Y, Cruz-Morales P, Zargar A, Belcher MS, Pang B, Englund E, Dan Q, Yin K, Keasling JD (2021) Biofuels for a sustainable future. Cell 184(6):1636-1647. https://doi.org/10.1016/j.cell.2021.01.052

Liu Z, Zheng W, Ye W, Wang C, Gao Y, Cui W, Zhou Z (2019) Characterization of cysteine sulfinic acid decarboxylase from Tribolium castaneum and its application in the production of beta-alanine. Appl Microbiol Biotechnol 103(23-24):9443-9453. https://doi.org/10.1007/s00253-019-10139-z

Ohara T, Sato T, Shimizu N, Prescher G, Schwind H, Weiberg O, Marten K, Greim H (2011) Acrylic Acid and Derivatives Ullmann's Encyclopedia of Industrial Chemistry.

Park JH, Lee SY (2010) Metabolic pathways and fermentative production of L-aspartate family amino acids. Biotechnol J 5(6):560-77. https://doi.org/10.1002/biot.201000032

Pei W, Zhang J, Deng S, Tigu F, Li Y, Li Q, Cai Z, Li Y (2017) Molecular engineering of L-aspartate-alphadecarboxylase for improved activity and catalytic stability. Appl Microbiol Biotechnol 101(15):6015-6021. https://doi.org/10.1007/s00253-017-8337-y

Piao X, Wang L, Lin B, Chen H, Liu W, Tao Y (2019) Metabolic engineering of Escherichia coli for production of L-aspartate and its derivative beta-alanine with high stoichiometric yield. Metab Eng 54:244-254. https://doi.org/10.1016/j.ymben.2019.04.012

Qian Y, Liu J, Song W, Chen X, Luo Q, Liu L (2018) Production of $\beta$-alanine from fumaric acid using a dual-enzyme cascade. ChemCatChem 10(21):4984-4991. https://doi.org/10.1002/cctc.201801050 
Sale C, Saunders B, Harris RC (2010) Effect of beta-alanine supplementation on muscle carnosine concentrations and exercise performance. Amino Acids 39(2):321-33. https://doi.org/10.1007/s00726009-0443-4

Shen Y, Zhao L, Li Y, Zhang L, Shi G (2014) Synthesis of $\beta$-alanine from l-aspartate using l-aspartate-adecarboxylase from Corynebacterium glutamicum. Biotechnology Letters 36(8):1681-1686. https://doi.org/10.1007/s10529-014-1527-0

Siripong W, Angela C, Tanapongpipat S, Runguphan W (2020) Metabolic engineering of Pichia pastoris for production of isopentanol (3-Methyl-1-butanol). Enzyme and Microbial Technology 138:109557. https://doi.org/10.1016/j.enzmictec.2020.109557

Siripong W, Wolf P, Kusumoputri TP, Downes JJ, Kocharin K, Tanapongpipat S, Runguphan W (2018) Metabolic engineering of Pichia pastoris for production of isobutanol and isobutyl acetate. Biotechnology for Biofuels 11(1). https://doi.org/10.1186/s13068-017-1003-x

Song CW, Lee J, Ko YS, Lee SY (2015) Metabolic engineering of Escherichia coli for the production of 3aminopropionic acid. Metab Eng 30:121-129. https://doi.org/10.1016/j.ymben.2015.05.005

Steunenberg P, Könst PM, Scott EL, Franssen MCR, Zuilhof H, Sanders JPM (2013) Polymerisation of $\beta$ alanine through catalytic ester-amide exchange. European Polymer Journal 49(7):1773-1781. https://doi.org/10.1016/j.eurpolymj.2013.03.032

Tan Z, Zhu X, Chen J, Li Q, Zhang X (2013) Activating phosphoenolpyruvate carboxylase and phosphoenolpyruvate carboxykinase in combination for improvement of succinate production. Appl Environ Microbiol 79(16):4838-44. https://doi.org/10.1128/AEM.00826-13

Tigu F, Zhang J, Liu G, Cai Z, Li Y (2018) A highly active pantothenate synthetase from Corynebacterium glutamicum enables the production of D-pantothenic acid with high productivity. Appl Microbiol Biotechnol 102(14):6039-6046. https://doi.org/10.1007/s00253-018-9017-2

Tomita H, Yokooji Y, Ishibashi T, Imanaka T, Atomi H (2014) An archaeal glutamate decarboxylase homolog functions as an aspartate decarboxylase and is involved in $\beta$-alanine and coenzyme $A$ biosynthesis. J Bacteriol 196(6):1222-30. https://doi.org/10.1128/jb.01327-13

Tuyishime P, Wang Y, Fan L, Zhang Q, Li Q, Zheng P, Sun J, Ma Y (2018) Engineering Corynebacterium glutamicum for methanol-dependent growth and glutamate production. Metab Eng 49:220-231. https://doi.org/10.1016/j.ymben.2018.07.011

Wang L, Piao X, Cui S, Hu M, Tao Y (2020) Enhanced production of beta-alanine through co-expressing two different subtypes of L-aspartate-alpha-decarboxylase. J Ind Microbiol Biotechnol 47(6-7):465-474. https://doi.org/10.1007/s10295-020-02285-5 
Weninger A, Fischer JE, Raschmanova H, Kniely C, Vogl T, Glieder A (2018) Expanding the CRISPR/Cas9 toolkit for Pichia pastoris with efficient donor integration and alternative resistance markers. J Cell Biochem 119(4):3183-3198. https://doi.org/10.1002/jcb.26474

Whitaker WB, Jones JA, Bennett RK, Gonzalez JE, Vernacchio VR, Collins SM, Palmer MA, Schmidt S, Antoniewicz MR, Koffas MA, Papoutsakis ET (2017) Engineering the biological conversion of methanol to specialty chemicals in Escherichia coli. Metab Eng 39:49-59.

https://doi.org/10.1016/j.ymben.2016.10.015

Wu C, Zhang J, Du G, Chen J (2013) Aspartate protects Lactobacillus casei against acid stress. Appl Microbiol Biotechnol 97(9):4083-93. https://doi.org/10.1007/s00253-012-4647-2

Yamada R, Ogura K, Kimoto Y, Ogino H (2019) Toward the construction of a technology platform for chemicals production from methanol: d-lactic acid production from methanol by an engineered yeast Pichia pastoris. World Journal of Microbiology and Biotechnology 35(2).

https://doi.org/10.1007/s11274-019-2610-4

Yu S, Miao L, Huang H, Li Y, Zhu T (2020a) High-level production of glucose oxidase in Pichia pastoris. Effects of Hac1p overexpression on cell physiology and enzyme expression. Enzyme Microb Technol 141:109671. https://doi.org/10.1016/j.enzmictec.2020.109671

Yu XJ, Huang CY, Xu XD, Chen H, Liang MJ, Xu ZX, Xu HX, Wang Z (2020b) Protein engineering of a pyridoxal-5'-phosphate-dependent l-aspartate-alpha-decarboxylase from Tribolium castaneum for betaalanine production. Molecules 25(6). https://doi.org/10.3390/molecules25061280

Zhu T, Sun H, Wang M, Li Y (2019) Pichia pastoris as a versatile cell factory for the production of industrial enzymes and chemicals: Current status and future perspectives. Biotechnol J 14(6):e1800694. https://doi.org/10.1002/biot.201800694

Zhu T, Zhao T, Bankefa OE, Li Y (2020) Engineering unnatural methylotrophic cell factories for methanolbased biomanufacturing: Challenges and opportunities. Biotechnol Adv 39:107467. https://doi.org/10.1016/j.biotechadv.2019.107467

Zhu WL, Cui JY, Cui LY, Liang WF, Yang S, Zhang C, Xing XH (2016) Bioconversion of methanol to valueadded mevalonate by engineered Methylobacterium extorquens AM1 containing an optimized mevalonate pathway. Appl Microbiol Biotechnol 100(5):2171-82. https://doi.org/10.1007/s00253-0157078-z

Zou X, Guo L, Huang L, Li M, Zhang S, Yang A, Zhang Y, Zhu L, Zhang H, Zhang J, Feng Z (2020) Pathway construction and metabolic engineering for fermentative production of beta-alanine in Escherichia coli. Appl Microbiol Biotechnol 104(6):2545-2559. https://doi.org/10.1007/s00253-020-10359-8

\section{Tables}


Table 1. Primers used in this study 
Primers name Sequences $\left(5^{\prime} \rightarrow 3^{\prime} \rrbracket\right.$

5'AOX1 GACTGGTTCCAATTGACAAGC

3'AOX1 GCAAATGGCATTCTGACATCC

His4-BamHI-F GCATGGATCCATGACATTTCCCTTGCTAC

His4-BamHI-R GCATGGATCCGTTAAATAAGTCCCAGTTTCTC

PMPICZA-3881-F GGTTTAGTTCCTCACCTTG

pMPICZA-4256-R TCCCCCTTTTCCTTTGTC

Kan-pMPICZA-F ACAAGGTGAGGAACTAAACCATGAGCCATATTCAACGG

Kan-pMPICZA-R TCGACAAAGGAAAAGGGGGATTAGAAAAACTCATCGAGC

Hyg-pMPICZA-F ACAAGGTGAGGAACTAAACCATGAAAAAGCCTGAACTCAC

Hyg-pMPICZA-R TCGACAAAGGAAAAGGGGGACTATTCCTTTGCCCTCGG

PTEF1-F GCAATCTAATCTAAGGGGCGGTGT

CYC1TT-R TTCGAGCGTCCCAAAACCTTCTCAAGC

ADCBs-Xhol-F GCATCTCGAGATGGATGTATCGAACAATGATGAGC

ADCBs-Notl-R GCATGCGGCCGCCTACAAAATTGTACGGGCTG

Oan-Xhol-F GCATCTCGAGATGTCCGTATCTGAAACC

Oan-Notl-R GCATGCGgCCGCTCAGATAACGGTAGTTGCTACGC

Spe-Xhol-F GCATCTCGAGATGAAAAAAATCATGATGATCGG

Spe-Notl-R GCATGCGGCCGCTTATGCGATAAAGCCACCGTC

AAT1-BstBI(gb)-F ATCAAAAAACAACTAATTATTCGAAATGTCGTTTTCAACGCAGAA

AAT1-Notl(gb)-R AGTTTTTGTTCTAGAAAGCTGGCGGCCGCCTATACACGCACAACCTGAT

AAT2-BstBI(gb)-F ATCAAAAAACAACTAATTATTCGAAATGTCGTTTTCAACGCAGAA

AAT2-Notl(gb)-R AGTTTTTGTTCTAGAAAGCTGGCGGCCGCCTATACACGCACAACCTGAT

AsPCK-BstBI(gb)-F ATCAAAAAACAACTAATTATTCGAAATGACTGACTTAAACAAACTCG

AsPCK-Notl(gb)-R AGTTTTTGTTCTAGAAAGCTGGCGGCCGCTTATGCTTTTGGACCGGCGCCAAC

PPC-BstBI(gb)-F ATCAAAAAACAACTAATTATTCGAAATGAACGAACAATATTCCGC

PPC-Notl(gb)-R AGTTTTTGTTCTAGAAAGCTGGCGGCCGCTTAGCCGGTATTACGCATAC

PCK-BstBI(gb)-F ATCAAAAAACAACTAATTATTCGAAATGCGCGTTAACAATGGTTTG

PCK-Notl(gb)-R AGTTTTTGTTCTAGAAAGCTGGCGGCCGCTTACAGTTTCGGACCAGCCGC 


\section{Figures}

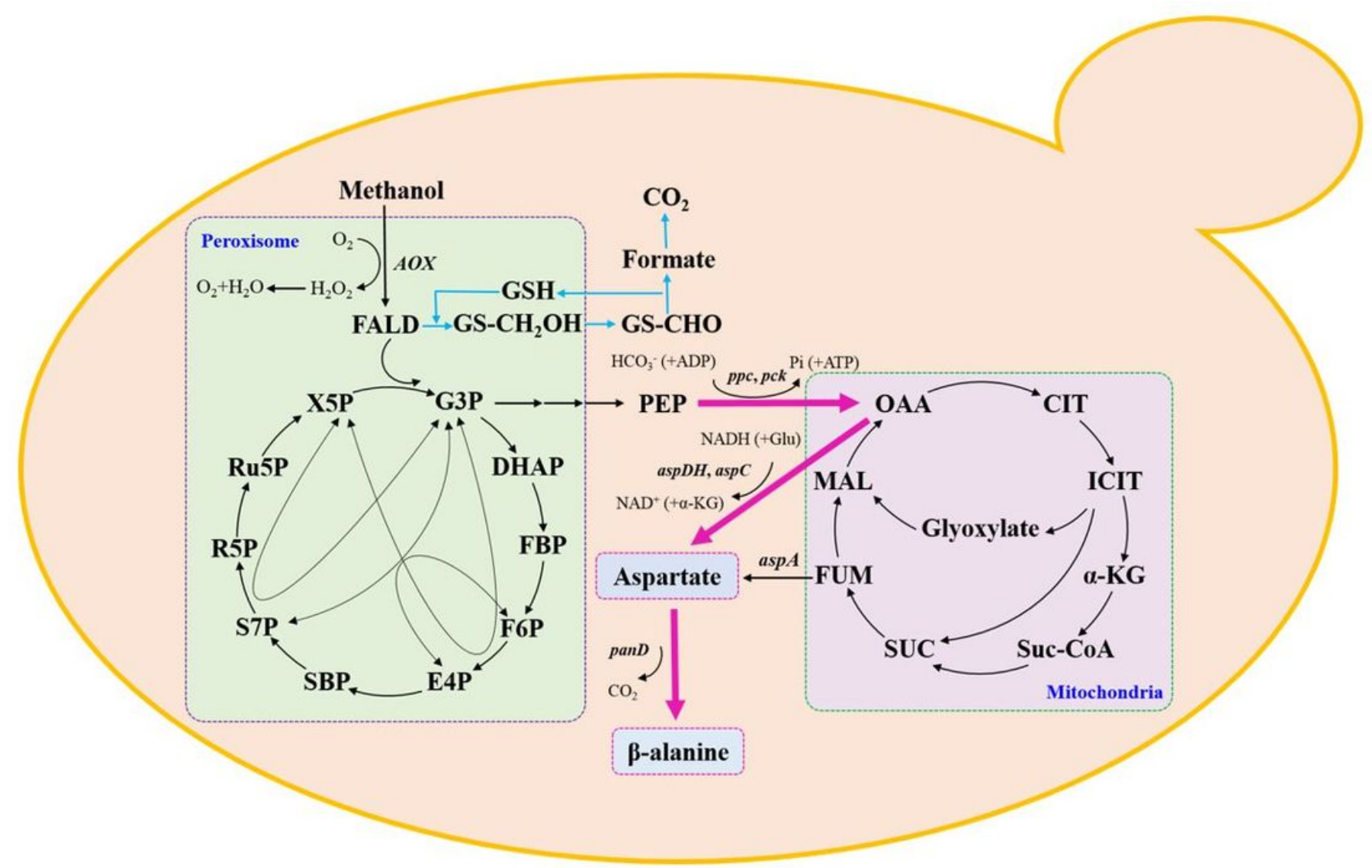

\section{Figure 1}

The biosynthesis pathway designed in P. pastoris for the production of $\beta$-alanine from methanol. Abbreviations: AOX: Gene encoding alcohol oxidase; ppc: Gene encoding PEP carboxylase; pck: Gene encoding PEP carboxykinase; aspDH: Gene encoding aspartate dehydrogenase; aspC: Gene encoding aspartate transferase; panD: Gene encoding aspartate-a-decarboxylases; FALD: Formaldehyde; X5P: Xylulose-5-phosphate; G3P: Glyceraldehyde-3-phosphate; DHAP: Dihydroxyacetone phosphate; FBP: Fructose-1,6-bisphosphate; F6P: Fructose-6-phosphate; E4P: Erythrose-4-phosphate; SBP: Sedoheptulose1,7-bisphosphate; S7P: Sedoheptulose-7-phosphate; R5P: Ribose-5-phosphate; Ru5P: Ribulose-5phosphate; PEP: Phosphoenolpyruvate; OAA: Oxaloacetate; CIT: Citrate; ICIT: Isocitrate; a-KG: aKetoglutarate; Suc-CoA: Succinyl-CoA; SUC: Succinate; FUM: Fumarate; MAL: Malate. 
Plasmids

\begin{tabular}{|c|c|c|}
\hline pPICZA & Vector for extracellular expression recombinant protein carrying $Z e o^{R}$ & Invitrogen \\
\hline $\mathrm{pAO}$ & His4+ & Invitrogen \\
\hline pMPICZHis & Extracellular expression vector carrying His4, Zeo ${ }^{R}$ & This work \\
\hline pMPIC & $\begin{array}{l}\text { Replace antibiotic resistance marker (zeocin) of pMPICZHis with } \\
\text { kanamycin }\end{array}$ & Thi \\
\hline
\end{tabular}

pMPIC2H

Replace antibiotic resistance marker (zeocin) of pMPICZHis with

This work hygromycin

pMPICZHis- Codon optimized panD gene from Bacillus paralicheniformis ATCC

This work

bliPanD 9945a cloned into pMPICZHis at BstBI and Notl site

pMPICZHisbsPanD

Codon optimized panD gene from Bacillus subtilis cloned into pMPICZHis at BstBI and Notl site

pMPICZHissgePanD

pMPICZHiscguPanD

pMPICZHisTCCSADC
Codon optimized panD gene from Streptomyces griseorubiginosus cloned into pMPICZHis at BstBI and Notl site

Codon optimized panD gene from Corynebacterium glutamicum cloned into pMPICZHis at BstBI and Notl site

Cysteine sulfinic acid decarboxylase from Tribolium castaneum (TCCSADC) cloned into into pMPICZHis at BstBI and Notl site

This work

This work

This work

This work

aspDH gene from Ochrobactrum anthropi ATCC 4918 cloned into

This work

pMPICZHis-

Oan pMPICZHis at BstBI and Notl site

pMPICZHisSpe

pMPICZHisADC-Oan

pMPICZHisADC-Spe

pMPICKmHisAAT1

pMPICKmHis-

AAT2

pMPICZHis-

2ADC

pMPICZHis-

2ADC-Spe

pMPIC2H-

PPC
aspDH gene from Serratia proteamaculanscloned into pMPICZHis at $B s t \mathrm{BI}$ and Notl site

panD expression cassette (pMPICZHis-ADC digest by Sphl and $B a m H I)$ cloned into pMPICZHis-Oan at Sphl and Bgfll site

panD expression cassette (pMPICZHis-ADC digest by Sphl and BamHI) cloned into pMPICZHis-Spe at Sphl and Bg/ll site

Cytosolic aspartate aminotransferase from P. pastoris GS115 cloned into pMPICZHis at BstBI and Notl site

Mitochondrial aspartate aminotransferase from P. pastoris GS115 cloned into pMPICZHis at BstBI and Notl site

Two copies of panD gene expression cassette cloned into pMPICZHis

Two copies of panD gene expression cassette and one copy of SpeaspDH gene expression cassette cloned into pMPICZHis

ppc gene from Escherichia colicloned into integration vector pMPIC2H at BstBI and Notl site
This work

This work

This work

This work

This work

This work

This work

This work 
pMPIC2H-

AsPCK

pMPIC2H-

PCK pck gene from Actinobacillus succinogenes cloned into integration vector pMPICHyHisLoxA at BstBI and Notl site

pck gene from Escherichia coli cloned into integration vector pMPIC2H at BstBI and Notl site
This work

This work

Strains

E. coli $\mathrm{DH} 5 \mathrm{a} \quad$ Commercial transformation host for cloning

Invitrogen

P. pastoris $\quad$ Commercial transformation host for cloning; his4- , Mut+ GS115

GS115, $\Delta k u 70 ;$ his4+

This work

$\Delta \mathrm{ku} 70$

$\triangle \mathrm{ku} 70$ harboring pMPICZHis-bliPanD

This work

BliADC

$\triangle \mathrm{ku} 70$ harboring pMPICZHis-bsPanD

This work

BsADC

$\triangle \mathrm{ku} 70$ harboring pMPICZHis-sgePanD

This work

SgeADC

$\triangle \mathrm{ku} 70$ harboring pMPICZHis-cguPanD

This work

CguADC

$\triangle \mathrm{ku} 70$ harboring pMPICZHis-TcCSADC

This work

ADC-Oan

$\triangle \mathrm{ku} 70$ harboring pMPICZHis-ADC-Oan

This work

ADC-Spe

$\triangle \mathrm{ku} 70$ harboring pMPICZHis-ADC-Spe

This work

ADC-AAT1

$\triangle \mathrm{ku} 70$ harboring pMPICZHis-bsPanD and pMPICKmHis-AAT1

This work

ADC-AAT2

$\triangle \mathrm{ku} 70$ harboring pMPICZHis-bsPanD and pMPICKmHis-AAT2

This work

2ADC

$\triangle \mathrm{ku} 70$ harboring pMPICZHis-2ADC

This work

2ADC-Spe

$\triangle \mathrm{ku} 70$ harboring pMPICZHis-2ADC-Spe

This work

PPC

$\triangle \mathrm{ku} 70$ harboring pMPICZHis-2ADC-Spe and pMPIC2H-PPC

This work

AsPCk

$\triangle \mathrm{ku} 70$ harboring pMPICZHis-2ADC-Spe and pMPIC2H-AsPCK

This work

PCK

This work 

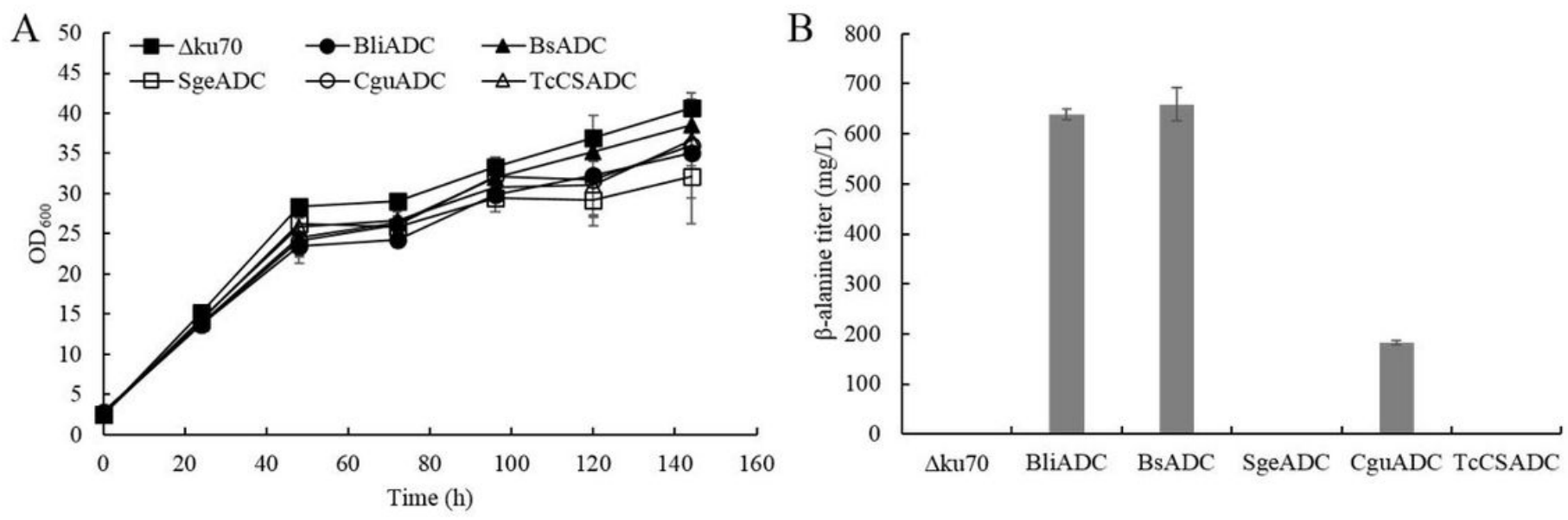

Figure 2

Overexpression of aspartate-a-decarboxylases (ADCs) from different sources for $\beta$-alanine synthesis. A. Growth profiles of recombinant strains; B. $\beta$-Alanine production in recombinant strains at the end of fermentation. Three parallel flasks are tested for each strain. Error bars represent deviations $(n=3)$.

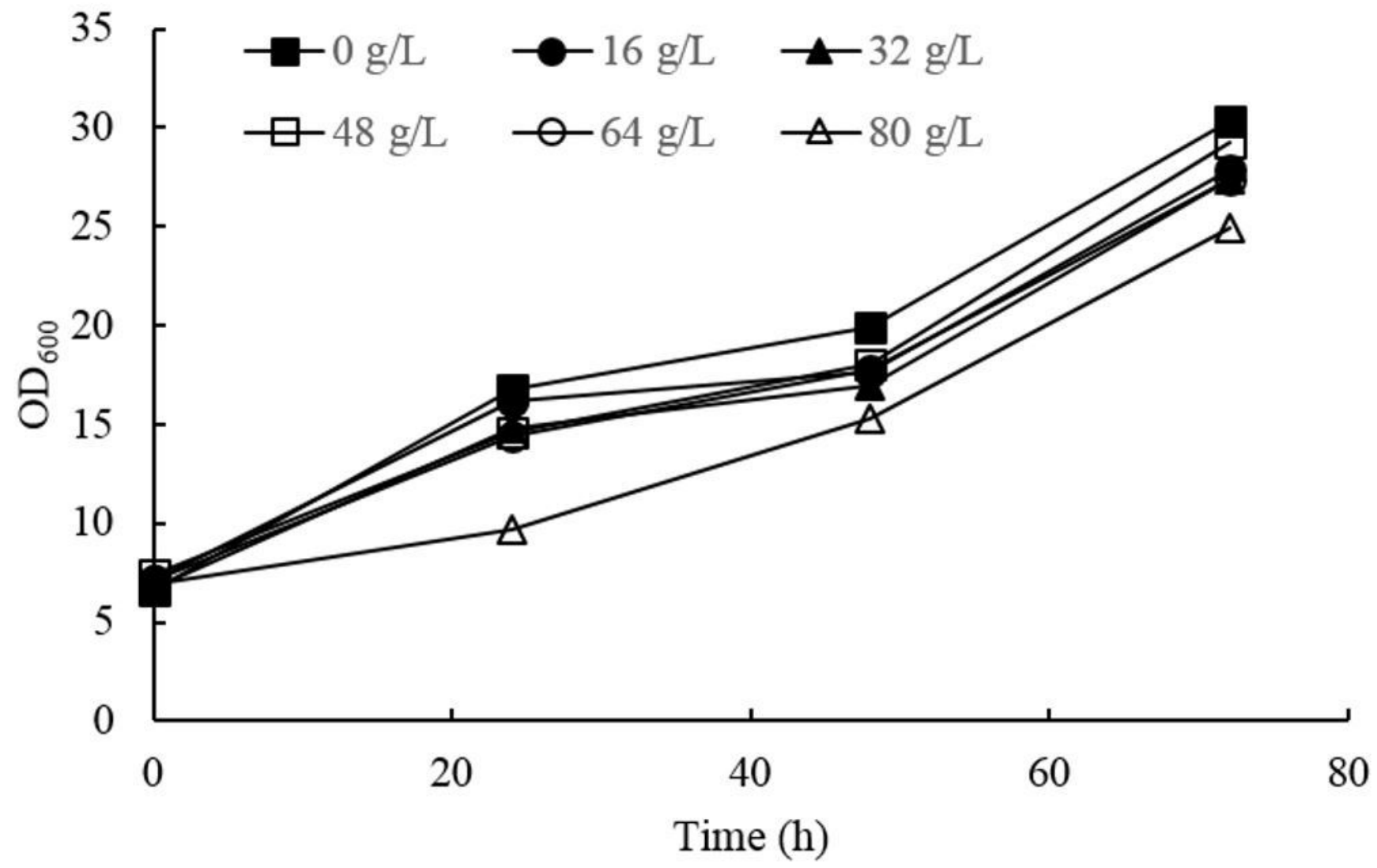

Figure 3

Tolerance of wild type P. pastoris strain for various concentrations of $\beta$-alanine. 

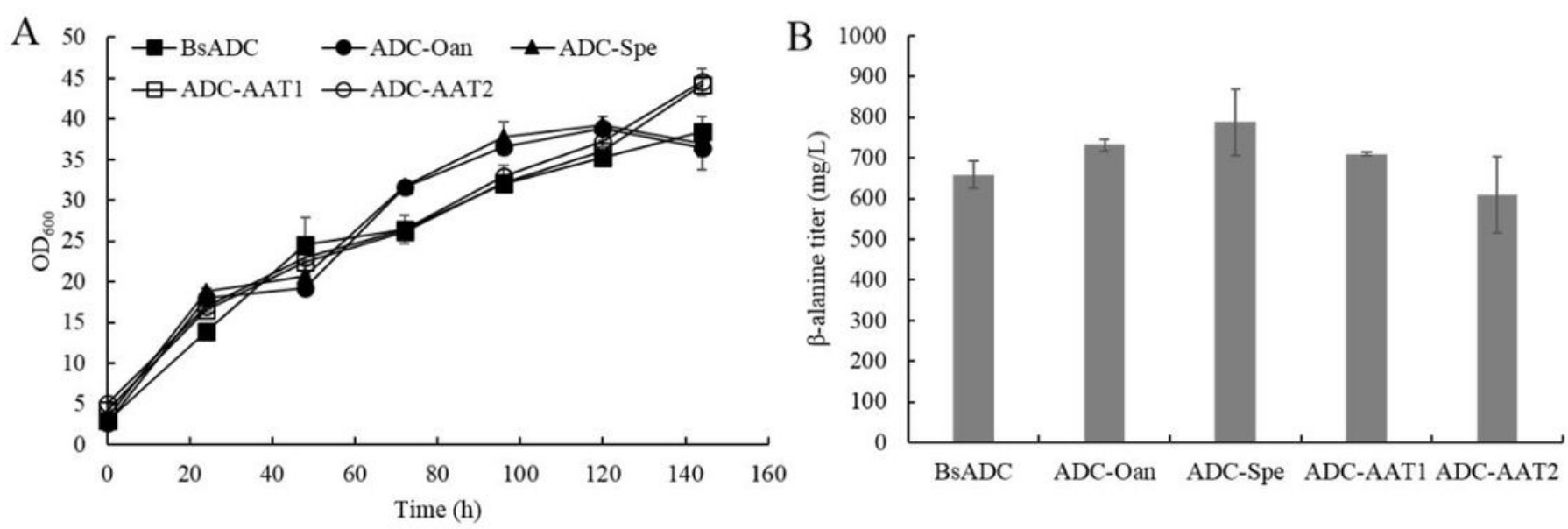

\section{Figure 4}

The effects of overexpression of aspartate transferase (AAT) or aspartate dehydrogenase (AspDH) on $\beta$ alanine production. A. Growth profiles of recombinant strains; B. $\beta$-Alanine production in recombinant strains at the end of fermentation. Three parallel flasks are tested for each strain. Error bars represent deviations $(n=3)$.
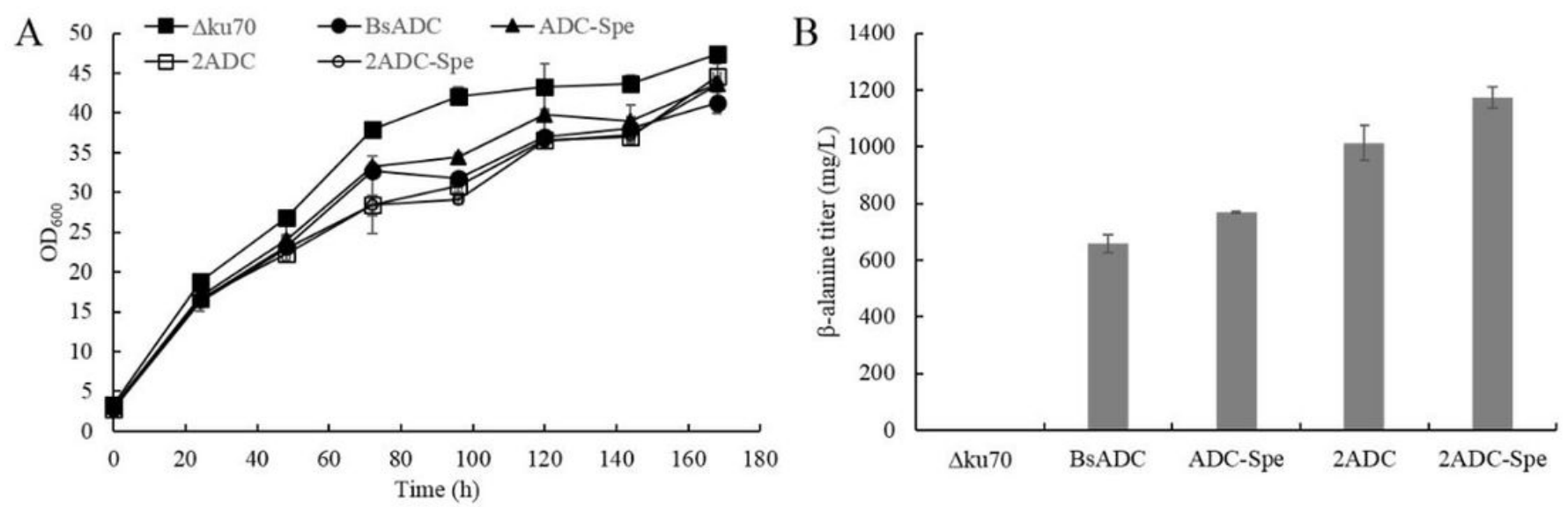

\section{Figure 5}

The effects of increasing ADC copy number on $\beta$-alanine production. A. Growth profiles of recombinant strains; B. $\beta$-Alanine production in recombinant strains at the end of fermentation. Three parallel flasks are tested for each strain. Error bars represent deviations $(n=3)$. 

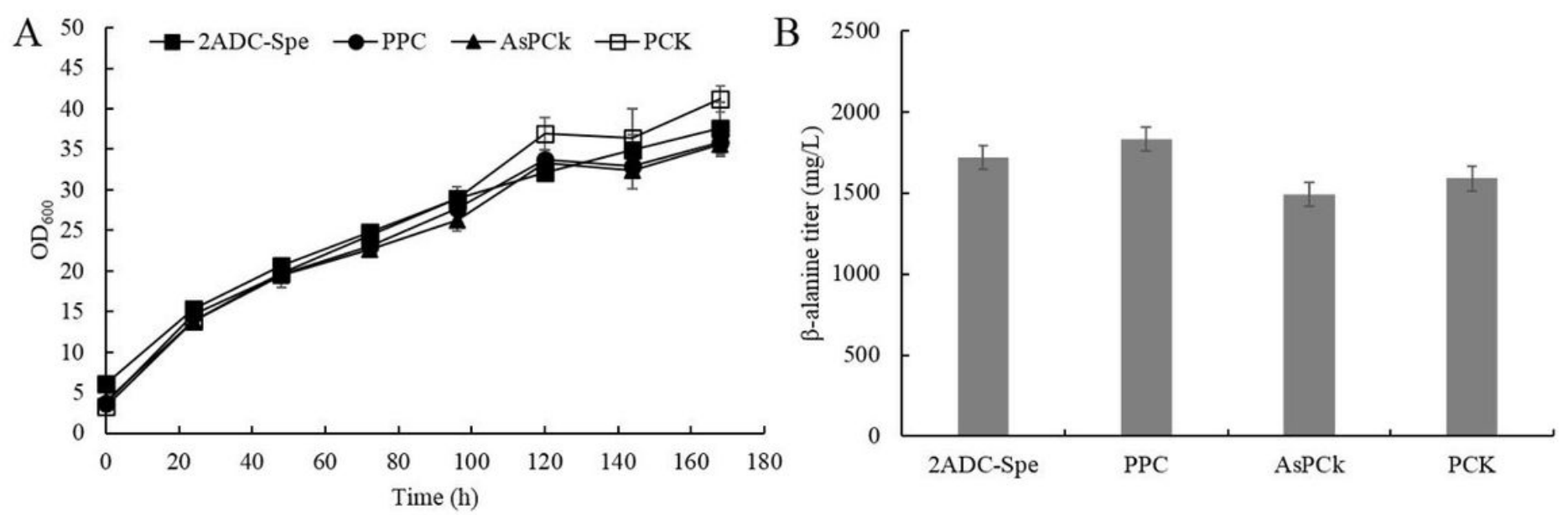

Figure 6

The effects of strengthening phosphoenolpyruvate (PEP) carboxylation on $\beta$-alanine production. $A$. Growth profiles of recombinant strains; B. $\beta$-Alanine production in recombinant strains at the end of fermentation. Three parallel flasks are tested for each strain. Error bars represent deviations $(n=3)$.

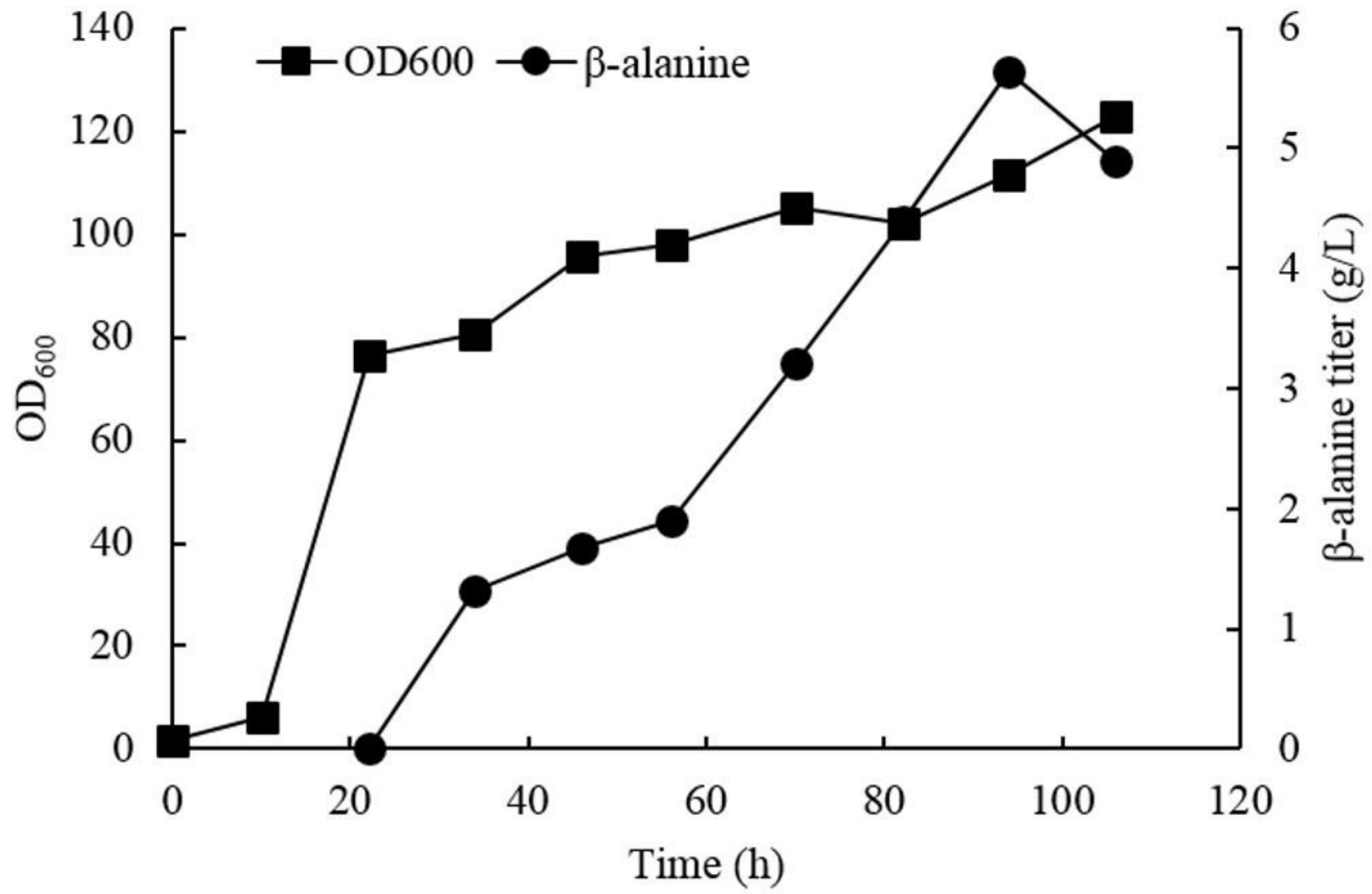

Figure 7 
Fed-batch fermentation profile of 2ADC-Spe strain.

\section{Supplementary Files}

This is a list of supplementary files associated with this preprint. Click to download.

- GraphicalAbstract.png 